\title{
Image enhancement for noisy color imagery*
}

\author{
Judith Dijk and Richard J.M. den Hollander \\ TNO Defence, Security and Safety \\ P.O. Box 96864, 2509 JG The Hague, The Netherlands \\ Email: Judith.Dijk@tno.nl
}

\begin{abstract}
Recently new techniques for night vision cameras are developed. So-called EMCCD cameras are able to record color information about the scene. However, in low-light situations this imagery becomes noisy. This is also the case for normal CCD cameras in dark situations or in shadowed areas. In this paper we present image enhancement techniques for noisy color imagery. The techniques are based on grey-value image enhancement techniques, in particular dynamic super-resolution reconstruction, which is used to enhance the lightness of the image, and local adaptive contrast enhancement. With the super-resolution technique the temporal noise in the lightness channel of the imagery is removed. The color information of the images is spatially filtered using the edge information of the enhanced lightness image. The result is colored output imagery with reduced temporal noise.
\end{abstract}

Keywords: image enhancement, noise reduction, super-resolution reconstruction, contrast enhancement, color EMCCD cameras

\section{INTRODUCTION}

For all operations situational awareness is of great importance. This situational awareness can be obtained by using cameras. In night-time, cameras usually record only grey-value information. This can be colored by using information from multiple cameras, such as for instance described by Toet. ${ }^{12}$ Currently, new camera techniques for night-time operations are being developed. With new-developed techniques such as EMCCD cameras, one can also record color images.

If these cameras are used in low light conditions, the resulting images may be rather noisy. Therefore, the need for noise reduction is high. Examples of images with different lighting conditions are shown in figure 1. One image is recorded inside our test facilities (figure 1(a)) and one outdoor (figure 1(b)). In both cases the usefulness of the images would increase if the noise is reduced without degrading the image information.

For grey value images such as IR imagery, algorithms have been developed for image enhancement, as for instance described by Schutte. ${ }^{9}$ One of these algorithms is noise reduction. This can be applied to the grey value channel of color images, after which the color of the original scene can be added. However, by adding the color information without any further steps, this will result in adding the noise in the color channels as well. Another image enhancement technique is contrast enhancement, which can also be applied on the grey value channel.

Parts of this research are described in European patent application No. 08152463.9 Luminance based edge preserved color smoothing

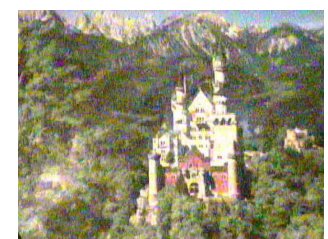

(a) castle (indoor)

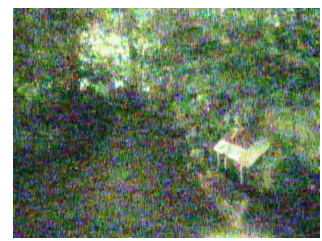

(b) bush (outdoor)

Figure 1. The test images. 
For color images, enhancement techniques such as super-resolution and de-mosaicking techniques are developed, for instance described by Battiato ${ }^{1}$ and Farsiu. ${ }^{4}$ In section 2 an overview of both grey value and color enhancement algorithms is given.

In this paper we apply existing grey value image enhancement techniques on color images. We present a new technique, in which temporal image enhancement is applied to the lightness image followed by a spatial color filtering. We also evaluate temporal image enhancement applied to the three color channels separately. Both techniques are compared with standard spatial color blurring. We also evaluate the effect of contrast enhancement in combination with these algorithms.

The proposed noise reduction technique reduces the noise in both the achromatic and chromatic signal in two subsequent steps. First we apply grey value noise reduction on the lightness channel using temporal information. Second, we apply an edge-dependent smoothing on the color channels. In contrast to normal edge preserving techniques the edge information is retrieved from the enhanced lightness image. So where the lightness is enhanced using temporal information, the color channels are smoothed using spatial information only. Note the similarity with the human eye, where the color is also filled-in using the grey value edge information. These techniques are compared with normal (spatial) edge preserving techniques.

This paper is organized as follows. In section 2 methods for noise reduction and contrast enhancement are discussed. In section 3 the tested algorithms are described, and in section 4 results for these algorithms are shown. In the last section the conclusions are given and issues for new research are discussed.

\section{LITERATURE}

\subsection{Noise reduction}

The removal of noise has been an active research topic in the past for both grey value and color images. Standard spatial filtering approaches exist for the removal of high frequencies, such as Gaussian smoothing. The major drawback of these approaches is that high frequency scene information, such as sharp edges, is also blurred.

To overcome this drawback, several methods have been developed which remove noise but also preserve the edges in the scene. Well-known examples are anisotropic diffusion ${ }^{8}$ and bilateral filtering. ${ }^{13}$ With anisotropic diffusion the amount of Gaussian diffusion is attenuated at the edges, by using an appropriate function of the gradient magnitude. The Gaussian diffusion is applied iteratively. For color images, the edge information is based on the achromatic signal such as the lightness or luminance signal.

In bilateral filtering a nonlinear combination of neighbouring pixels is used to smooth the image while preserving the edges. For each pixel in the image, the weights of the neighbouring pixels depend both on the similarity to the current pixel as on the distance to this pixel. On color images the filter is usually applied on the CIELAB color space, where the edge information is based on the lightness signal. In this way colors are smoothed and edges are preserved. Tomasi claims that with bilateral filtering no new colors will appear along the edges. An advantage of the bilateral filtering approach is that it is faster than anisotropic diffusion, because the method is non-iterative. In the proposed algorithm we base the similarity of the pixel on the difference with the enhanced lightness signal instead of the original lightness signal.

In case multiple frames of a scene are available temporal filtering can produce better results. When the camera and scene is stationary or the frames can be aligned accurately, temporal filtering will not deteriorate the scene structure as spatial filtering does. Temporal noise reduction can be done using averaging of the aligned frames, or using more complex techniques such as super resolution. An overview of such techniques is given by Park. ${ }^{7}$ Schutte et.al ${ }^{9}$ presented the Dynamic Super Resolution algorithm, which can be implemented in real-time. This algorithm can also be used for noise reduction, that is for enhancement without increasing the number of pixels. One of the challenges of temporal noise reduction is the preservation of moving elements in the scene.

There are two "intuitive" ways of performing super-resolution on color images using grey-value algorithms. The first is to apply the super-resolution algorithm to the three color planes R, G and B independently. However, this may change the ratio between the RGB values of pixels, and therefore unnatural colors and colored borders

will appear. Another method is to apply the super-resolution algorithm only to a grey-value channel, such as luminance or lightness. The output color image is then obtained by adjusting the color according to the grey 
value change. The fact that super-resolution is not applied on the color channels is not really a problem, as humans are not so sensitive to high frequency color changes.

Farsiu ${ }^{4}$ also developed color super-resolution techniques, which can be applied on normal and Bayered color images. These techniques have as advantage that all information in the images is used, and that they can be applied on Bayered color images. Another advantage is that no new colors will appear along the edges. These techniques are not evaluated in this paper.

\subsection{Local contrast enhancement}

Next to noise reduction, a grey value image can also be enhanced using contrast enhancement. The goal of such enhancement is to visualise more details in the image. To enhance the contrast in an image several methods are described in literature. The most simple way to enhance the contrast is by adjusting the image to the available range, so-called global contrast stretching. This will help in cases were only part of the available range is used, but will fail in situations were the range used in one part of the image is very different than in another. To enhance the contrast in a part of the available contrast range gamma manipulation can be done. In this case details in part of the lightness range of the image will be enhanced, at the cost of decreasing the lightness in other parts of the image. Another global method is histogram equalization, were the lightness values are changed so that the histogram of the image are as flat as possible. The main disadvantage of this method is that the output images are not so natural anymore.

To enhance local contrasts also local adaptive contrast enhancement can be used. The idea of local contrast enhancement is that the processing depends features in a local region. Narenda and Fitch ${ }^{6}$ propose a method in which the local statistics are described by its local mean and variance. Using a local region means that the contrast is enhanced in a specific scale in the image, for instance only small details. However, it is hard to decide which details are important and which are not. Most authors suggest therefore that one the contrast should be adjusted at several scales in the image. To do so, either different scales can be determined or a multi-scale pyramid can be constructed.

In our evaluation we use LACE, a grey-value algorithm ${ }^{10}$ which is based on the method of Narenda and Finch, but extended to a number of scales. The output image $o$ is derived by

$$
o=i+\sum_{j=1}^{k} c\left(m_{j}, s_{j}\right)\left(i-m_{j}\right)
$$

where $m_{j}$ and $s_{j}$ are the local mean and standard deviation at scale $j . k$ is the total number of scales. The contrast enhancement function at each scale is given by

$$
c\left(m_{j}, s_{j}\right)=\alpha \frac{m_{j}}{s_{j}}-1
$$

where $\alpha$ is the weighing factor with the original image. $c\left(m_{j}, s_{j}\right)$ is clipped to $A_{\max }$ on top and zero on the bottom of the range.

This algorithm can be used to obtain images showing all interesting features in an image. An advantage of this algorithm is that it can be implemented on hardware, as well in a real-time software application. Dijk et.al ${ }^{3}$ proposed to perform the local contrast enhancement on color images by performing the contrast enhancement on the luminance channel, after which we recombine it with its colors.

\section{ALGORITHM}

In this section the algorithms that are tested are described. We test the effects of two enhancement techniques: noise reduction and contrast enhancement. As noise reduction technique we use the Dynamic Super Resolution (DSR) technique. ${ }^{9}$ For contrast enhancement we apply the Local Adaptive Contrast Enhancement technique (LACE). ${ }^{10}$ However, other noise reduction and contrast enhancement techniques could be used as well.

We evaluate what improvement can be found when applying these techniques on color images. We start by applying the DSR algorithm on the RGB channels separately. LACE can be applied on the RGB channels 


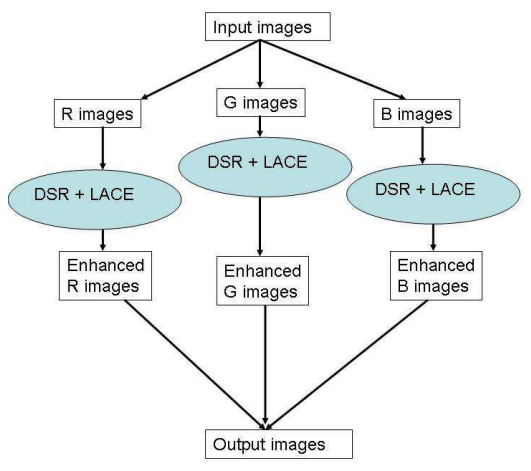

(a)

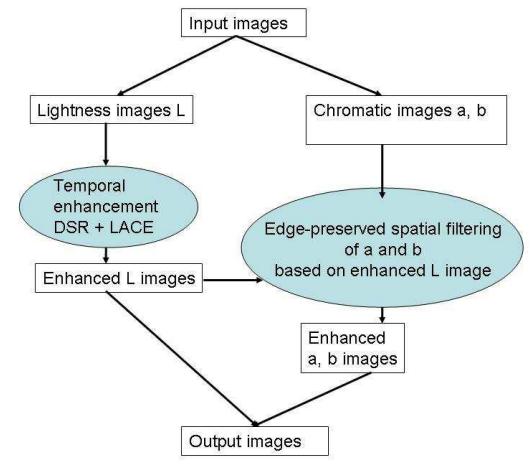

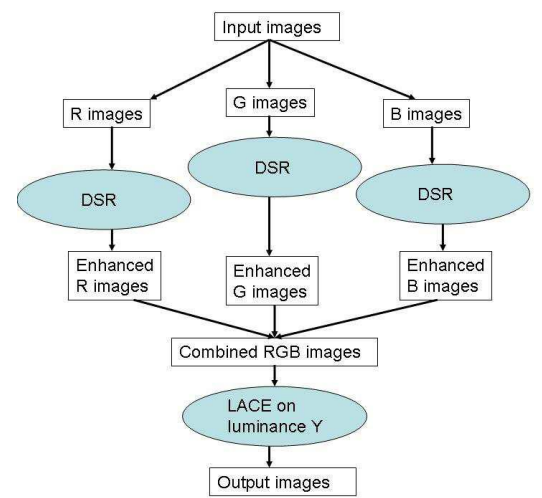

(b)

(c)

Figure 2. Schematic overview of processing steps. The processing is explained in the text.

directly, or on the combined luminance image. The enhanced R, G and B images are combined in one enhanced RGB image. LACE can be applied on the R, G, and B channels individually, or on the luminance of the combined image. These processing steps are visualised in figure 2 (a) and (b), respectively.

The second method is a new method we propose in this paper. Here we apply the enhancement on the lightness and color signals from the CIELAB space. The lightness signal is enhanced using the DSR algorithm. The color signals are spatially enhanced with edge preserving techniques. In contrast to standard edge preserving techniques, the edge information is retrieved from the enhanced lightness image instead of the original lightness image. LACE can then be applied on the enhanced lightness image.

This idea is visualised in figure 2 (c). First the images are split into an achromatic and a chromatic signal. In this paper we use the CIELAB color space, but other color spaces such as CIEXYZ or HSV can also be used. The achromatic lightness signal is enhanced using standard grey-value temporal enhancement techniques. The enhancement can be done on the lightness signal ( $\mathrm{L}$ from CIELAB), but also on the luminance ( $\mathrm{Y}$ from CIEXYZ). Then the color images $a^{*}$ and $b^{*}$ are enhanced using an adapted edge preserving technique. Similar to standard edge preserving techniques the smoothing of the pixel depends on two variables: the intensity difference with neighboring pixels and their distance to this pixel. The difference with standard edge preserving techniques is that the edge information is determined based on the enhanced achromatic image.

The rationale for this processing is that composing a lightness image from an RGB image will give an image 
with less noise, as the R.G and B realizations of one frame are combined into one lightness image. The achromatic images should be enhanced while keeping the high frequencies, as humans are more sensitive to high frequencies in the achromatic signal.

Humans are not sensitive to high frequency color changes, therefore the colors can be enhanced in the spatial domain. However, the blurring of the color signals should not cross the luminance or lightness changes. Therefore, the blurring should be within one area of the luminance or lightness image, and should not cross the borders.

In this paper we show results for bilateral filtering and median filtering as edge preserving algorithm, but other techniques such as anisotropic diffusion can also be used.

\section{RESULTS}

In this section we show the results for the different algorithms applied on the two input images shown in figure 1. The first test image, figure 1(a) is a frame from a recording in our test facility. In this image a postcard with a castle is seen. The luminance of the lamp at the position of the post card is 0.1 lux. The second image, figure 1(b), is an image from a movie recorded in the zoo in Arnhem in a bush environment. Both images are recorded with a Toshiba IK 1000 Low light EM-CCD NTSC Color Camera. In figure 3 and 4 the RGB input planes of the images are shown. Especially in the B plane can be seen that a large amount of compression is applied, because the noise is smeared over blocks. This compression hinders the spatial blurring of the colors, because the compressed noise is spatial correlated.

In figure 5 the results of temporal enhancement of the lightness channel of the castle image are shown. It can be seen that the enhanced image contains less noise than the input lightness image. Also, applying LACE improves the contrast in the image.

In figure 6-8 the results for the color castle image are shown. In figure 6 temporal enhancement of 6 the RGB images is applied. In figure 6(a) the input image is shown for comparison. In 6(b) the results for temporal enhancing the RGB channels separately is shown. It can be seen that the color noise is almost removed. Some color noise can still be seen as colored dots in the image. Applying LACE on the RGB channels as shown in 6(c) increases the contrast, but also changes the appearance of the image. The contrast enhancement increases the color noise, as expected. Applying the contrast enhancement on the luminance channel of the RGB image, as proposed by Dijk et.al., ${ }^{3}$ increases the contrast without changing the appearance very much. This result is shown in $6(\mathrm{~d})$.

In figure 7 the results for bilateral filtering are shown. In (b) standard bilateral filtering is applied. It can be seen that small details, such as the windows, are lost, and the noise is still visible. In (c) bilateral filtering based on the enhanced lightness image is shown. It can be seen that the small details are still visible. The color noise is still slightly present, due to the compression of the input images. In contrast to the RGB enhancement, this noise is blurred and less localized. We assume that using uncompressed recordings, this color noise will be less visible. The same LACE algorithm as in $6(\mathrm{~d})$ is applied on the lightness image. The result is shown in figure $7(\mathrm{~d})$. It can be seen that the appearance of the image is the same as the original image, and the contrast in the image is increased.

In figure 8 the results for median filtering are shown. In (b) standard median filtering on the lightness signal and the color signals $\mathrm{a}$ and $\mathrm{b}$ is applied. The size of the window is $3 \mathrm{x} 3$. It can be seen details are lost. In (c) median filtering of the $\mathrm{a}$ and $\mathrm{b}$ channel based on the enhanced lightness image is shown. The size of the window is $15 x 15$. It can be seen that the small details are still visible. The color noise is still slightly present, due to the compression of the input images. The LACE result is shown in figure 7(d). As was also seen for the bilateral filtering, applying LACE on the lightness image increases the contrast while keeping the appearance similar.

For the bush image the results are shown in figure 9-12. These results are comparable to the results for the castle image. One image is different, the RGB enhanced with LACE applied on the luminance (figure 10(d)). Here the effect of LACE is that the image is more or less black-and-white. 


\section{CONCLUSIONS AND DISCUSSION}

In this paper we present a new method for image enhancement of noisy color images. This method is performed in two steps. First a grey-value noise reduction based on temporal differences is applied on the lightness component. The color channels are spatially filtered using edge-preserving filtering techniques, where the edges are obtained from the enhanced lightness image. Also, applying temporal image enhancement on the RGB color channels separately is studied. Results show that both techniques are promising for low-light level color images. As expected, for RGB filtering some false colors can occur in the combined image. This is even more visible when contrast enhancement is applied on the R, G and B channels separately. The edge preserved filtering of the color images based on the enhanced lightness signal is a promising technique. In the results shown here some residual color noise is visible, but we assume that these are due to the compression which is applied the images. Therefore, for new recordings we should take care that no compression is used.

An advantage of the proposed technique, is that the temporal noise reduction technique is only applied once. As this is an expensive technique, we assume that the edge-preserving filtering can be implemented faster. In next studies we will address the effects of compression on the spatial filtering results, and also study how well color super-resolution techniques can be used for noise reduction for this kind of images.

\section{REFERENCES}

1. Battiato S., G. Gallo, F. Stanco , Smart Interpolation by Anisotropic Diffusion Proc. of ICIAP03, 17-19 Sept. 2003 pp 572- 577, 2003

2. Dijk, Judith (2004) In search of an Objective Measure for the Perceptual Quality of Printed Images PhD thesis, Delft University of Technology

3. Dijk, Judith, Richard J.M. den Hollander, John G.M. Schavemaker and Klamer Schutte (2007) Local Adaptive Contrast Enhancement for Color Images Proc. of SPIE, Vol. 6575, 2007

4. Farsiu,Sina, Dirk Robinson, Michael Elad, Peyman Milanfar, Advances and Challenges in Super-Resolution, International Journal of Imaging Systems and Technology Vol. 14, 4757, 2004

5. Florent, R. Patent USP nr. 6204891, USA, 2001.

6. Narenda, P.M. and R.C. Finch Real-time adaptive contrast enhancement IEEE transactions on pattern analysis and machine intelligence vol. 3, no. 6, pp 655-661, 1981

7. Sung Cheol Park, Min Kyu Park and Moon Gi Kang, Super- Resolution Image Reconstruction: A Technical OverviewIEEE Signal Processing Magazine, pages 21-36, 2003.

8. Perona, P., J. Malik, "Scale-Space and Edge Detection Using Anisotropic Diffusion," IEEE Transactions on Pattern Analysis and Machine Intelligence, vol. 12, no. 7, pp. 629-639, Jul., 1990

9. K. Schutte, D. de Lange, and S. van den Broek, "Signal conditioning algorithms for enhanced tactical sensor imagery," in SPIE proceedings: Infrared Imaging Systems: Design, Analysis, Modeling, and Testing XIV, 5076, pp. 92-100, 2003.

10. Schutte, K. Multi-scale adaptive Gain control of IR images SPIE vol 3061, pp.906-914, 1997

11. Shimoni, Y. Patent USP nr. 5049995, Israel, 1991.

12. Toet, A. Applying daytime colors to multiband nightvision imagery. Proceedings of the Sixth International Conference on Information Fusion (FUSION 2003). Fairborn, OH, USA: International Society of Information Fusion, , $614-621,2003$

13. Tomasi C. and R. Manduchi. Bilateral filtering for gray and color images. Proc. of ICCV, 1998. 


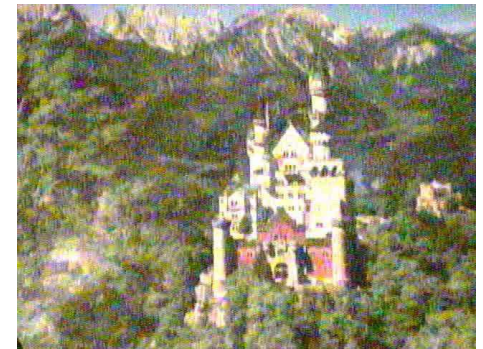

(a) RBG input image

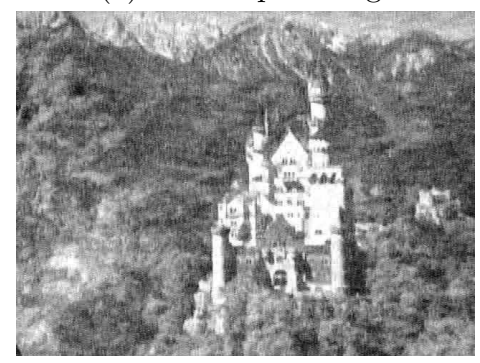

(c) input G image

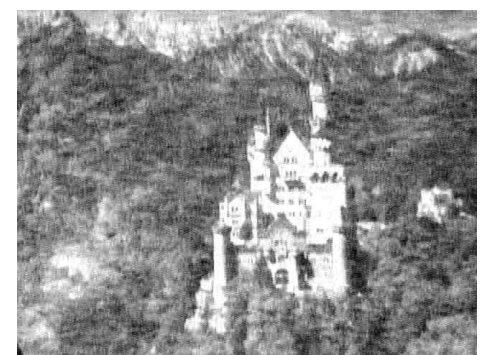

(b) input $\mathrm{R}$ image

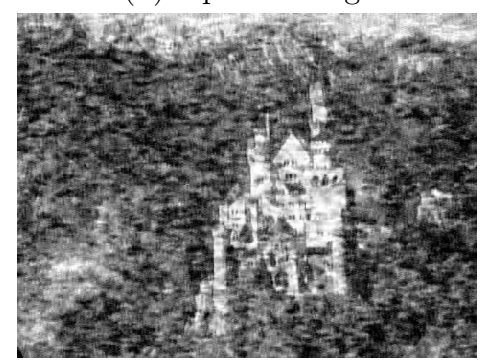

(d) input B image

Figure 3. Input (Castle).

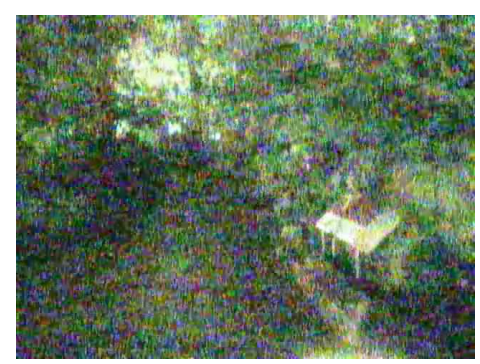

(a) input color image

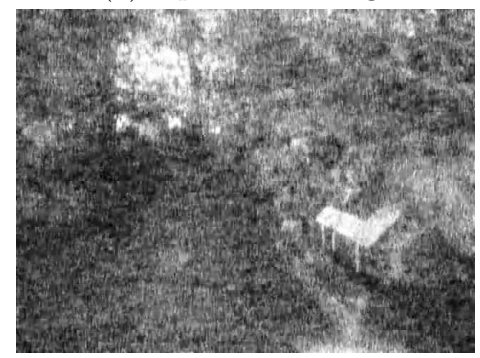

(c) input G image

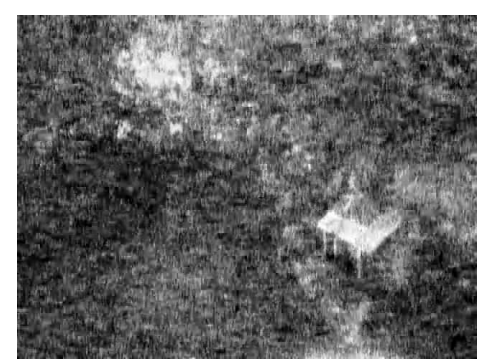

(b) input $\mathrm{R}$ image

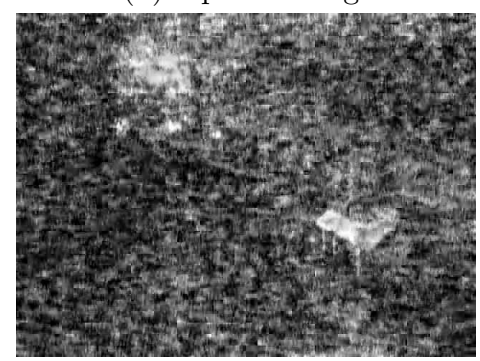

(d) input B image

Figure 4. Input (Bush) 


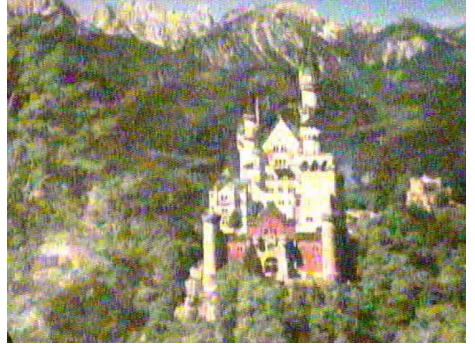

(a) input color image

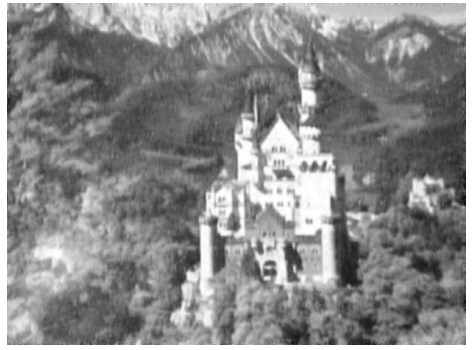

(c) enhanced lightness image

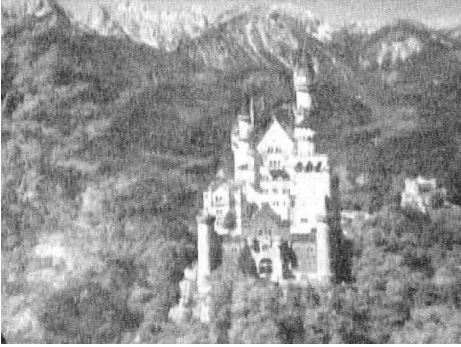

(b) input lightness image

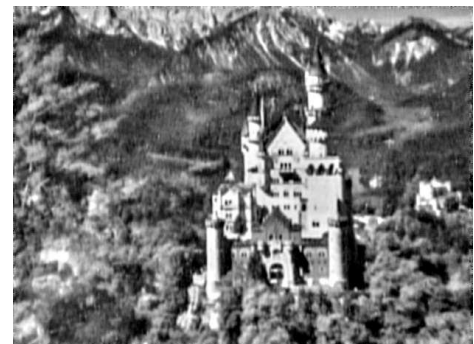

enhanced lightness image with LACE

Figure 5. Temporal lightness enhancement (Castle).

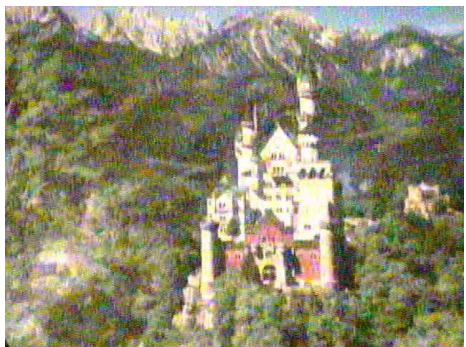

(a) input color image

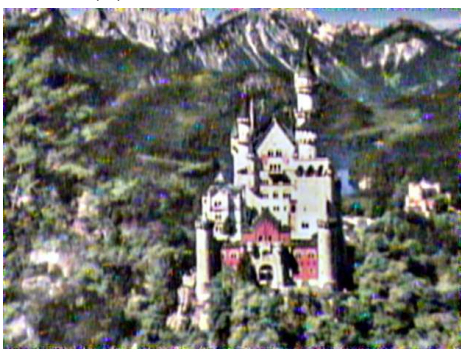

(c) RGB enhanced + LACE

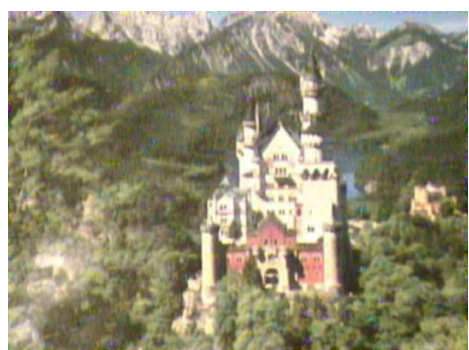

(b) RGB enhanced

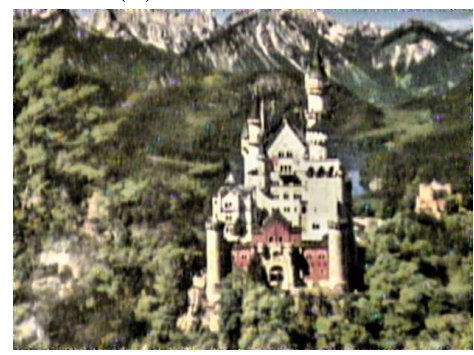

(d) RGB enhanced + LACE on luminance

Figure 6. Temporal enhancement of RGB channels (Castle). 


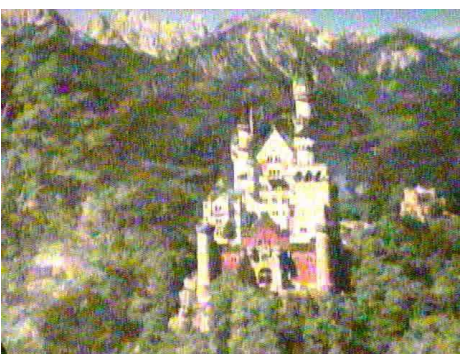

a) input color image

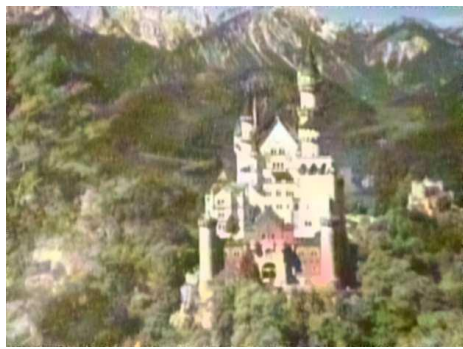

(c) using enhanced lightness edges

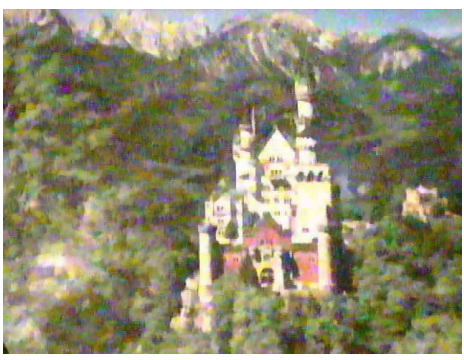

(b) bilateral filtering

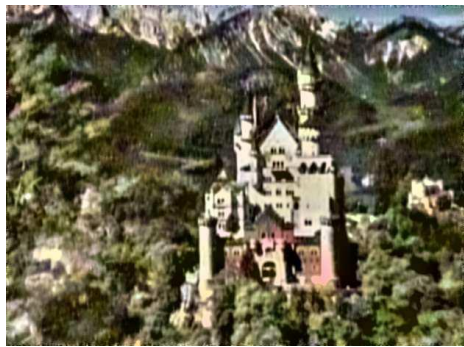

(d) using enhanced (with LACE) lightness edges

Figure 7. Bilateral filtering (Castle).

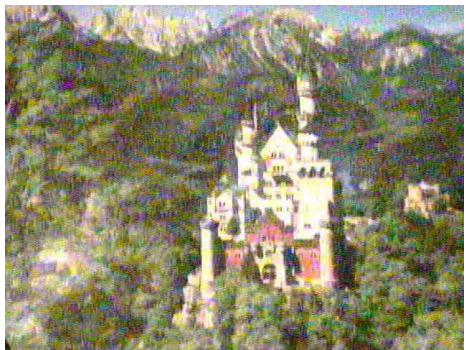

(a) input color image

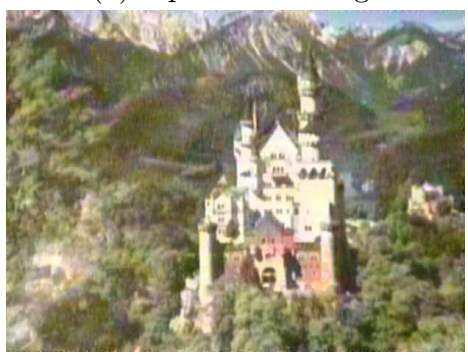

(c) using enhanced lightness edges

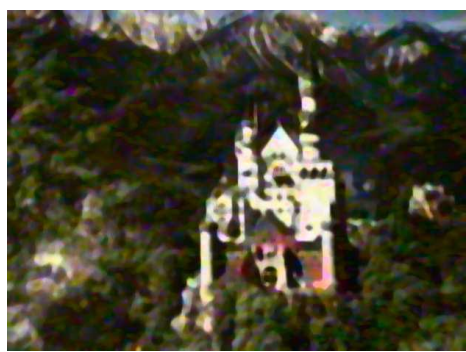

(b) standard median filtering

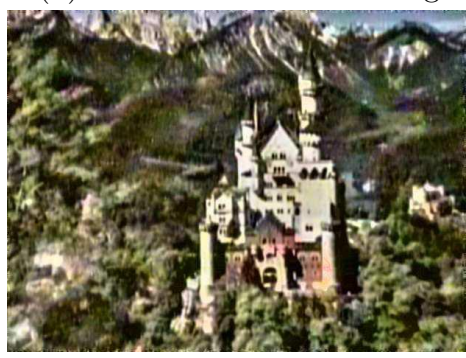

(d) using enhanced (with LACE) lightness edges

Figure 8. Median filtering (Castle). 


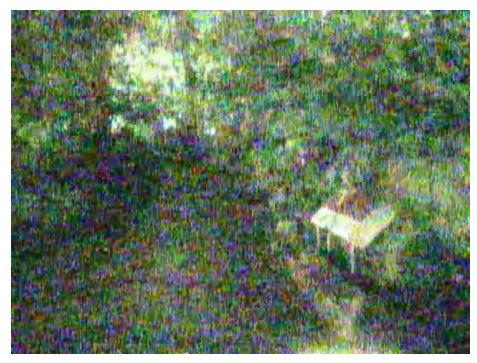

(a) input color image

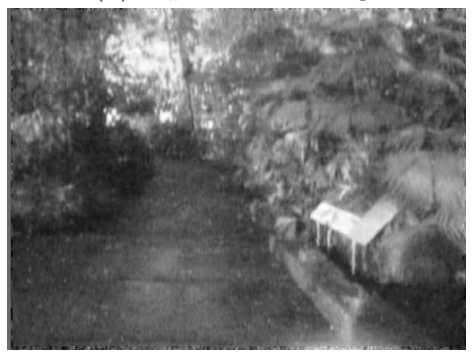

(c) enhanced lightness image

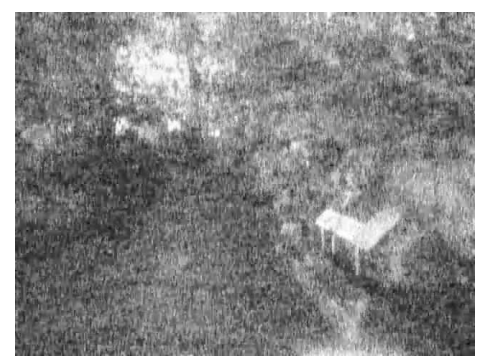

(b) input lightness image

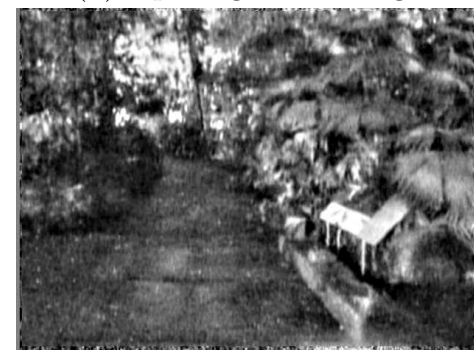

enhanced lightness image with LACE

Figure 9. Temporal lightness enhancement (Bush).

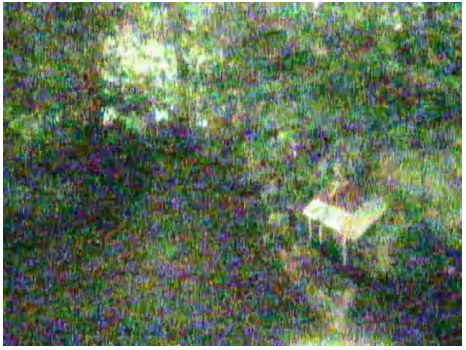

(a) input color image

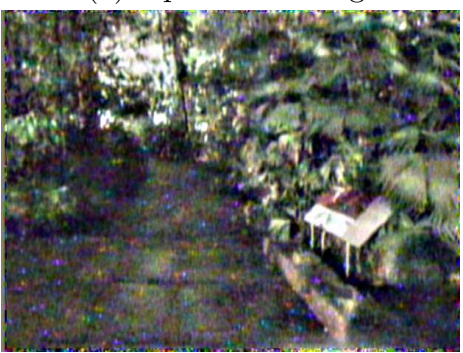

RGB enhanced + LACE

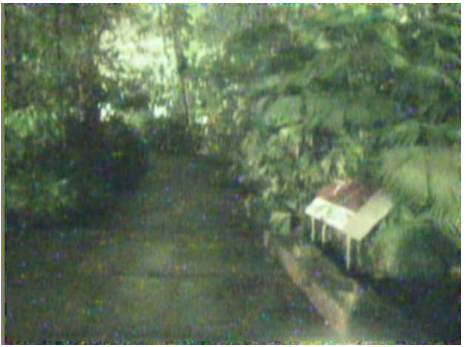

(b) RGB enhanced

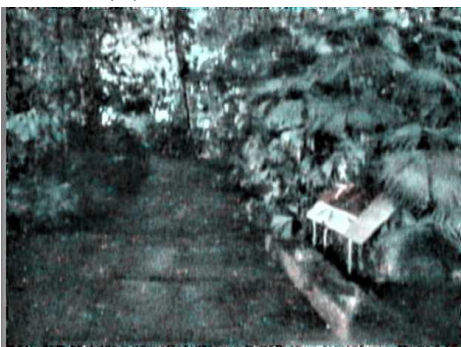

(d) RGB enhanced + LACE on luminance

Figure 10. Temporal enhancement of RGB channels (Bush). 


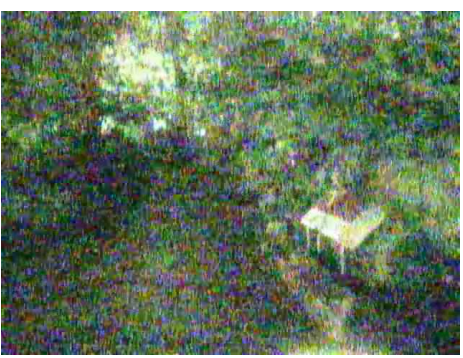

(a) input color image

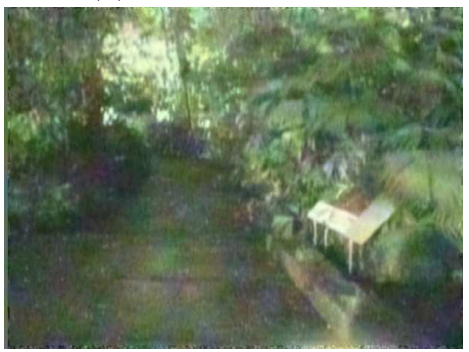

(c) using enhanced lightness edges

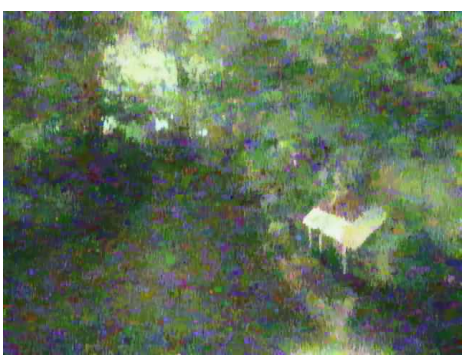

(b) bilateral filtering

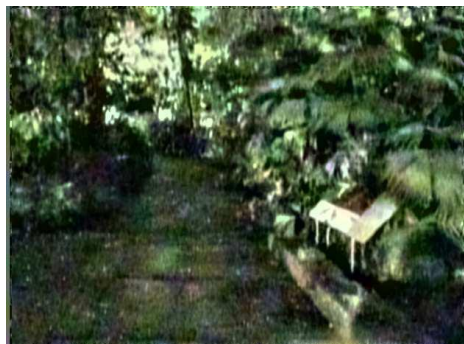

(d) using enhanced (with LACE) lightness edges

Figure 11. Bilateral filtering (Bush).

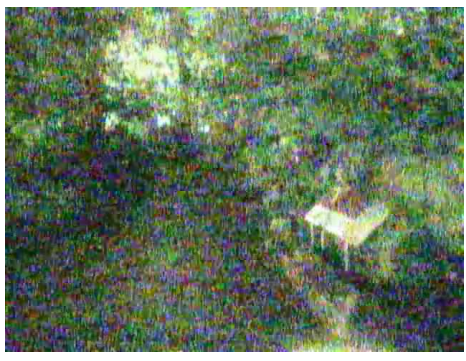

(a) input color image

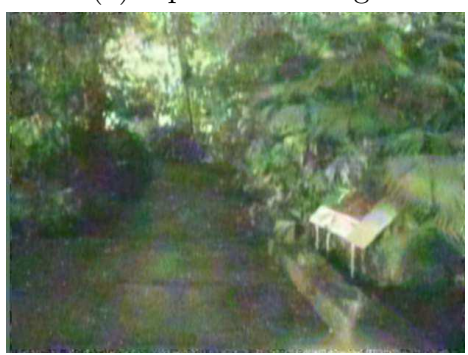

(c) using enhanced lightness edges

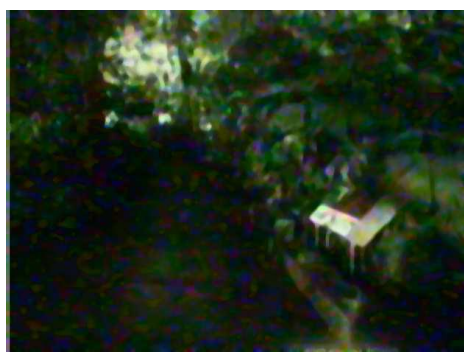

(b) standard median filtering

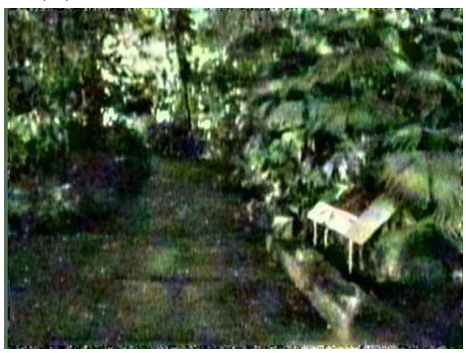

(d) using enhanced (with LACE) lightness edges

Figure 12. Median filtering (Bush). 